\title{
A new delirium phenotype with rapid high amplitude onset and nearly as rapid reversal: Central Coast Australia Delirium Intervention Study
}

This article was published in the following Dove Press journal:

Clinical Interventions in Aging

13 February 2015

Number of times this article has been viewed

\section{Paul J Regal}

Geriatric Medicine, University of Newcastle, Callaghan, Australia
Correspondence: Paul J Regal PO Box 105, Wyong, NSW

2259 Australia

Tel +6 I 24392525 I

Fax +6I 243935268

Email regalelderlymedicine@gmail.com
Background: Traditional models for delirium based on the Diagnostic and Statistical Manual for Mental Disorders and its 1990 offspring, the Confusion Assessment Method (CAM), were not designed to distinguish behavioral and psychological symptoms of dementia from rapid cognitive decline. We examined a new diagnostic criterion for delirium plus exclusion of behavioral and psychological symptoms of dementia and recent inattention with a $25 \%$ decline in digit span forward (DSF).

Methods: This was a prospective, randomized controlled trial comparing management of prevalent delirium in general medical with that in geriatric medical wards in a 370-bed hospital north of Sydney. Inclusion criteria were age $\geq 65$ years and prevalent delirium in the emergency department based on: CAM; proof that CAM elements were not better explained by behavioral and psychological symptoms of dementia; proof of recent inattention on DSF; evidence of cognitive decline not due to sedatives or antipsychotics in the emergency department. Measurements included the Instrumental Activities of Daily Living (IADL, 22-item), Selective IADL (8-item), Mini-Mental State Examination, DSF daily, Delirium Index daily, and Apathy Evaluation Scale. Pre-delirium scores from past cognitive tests and best scores were imputed after admission. Relative change (RC) was calculated as absolute change/test range and $\mathrm{RC} / \mathrm{MPC}$ ratio was calculated as $\mathrm{RC}$ after admission/maximal possible change.

Results: A total of 130 subjects were recruited but 14 with subsyndromal delirium were excluded, leaving 116 subjects (mean age 83.6 years). Forty-eight percent had prior dementia. RC from pre-delirium to admission was $42 \%$ for the Mini-Mental State Examination, $41 \%$ for Selective IADL, 34\% for 5-DSF, 54\% for 6-DSF, and 37\% for the Apathy Evaluation Scale. Improvements after admission (RC and RC/MPC ratios) were 32\%/98\% for 5-DSF, 54\%/82\% for $6-\mathrm{DSF}$, and $45 \% / 80 \%$ for the Delirium Index. General medicine and geriatric medicine groups had similar outcomes.

Conclusion: This delirium phenotype selects for a rapid high amplitude critical decline in attention, executive function, IADL, and apathy that recovers almost as rapidly.

Keyword: delirium, inattention, executive function, dementia

\section{Introduction}

Provenance refers to place of origin or earliest known time of appearance. Although provenance is typically used to trace precious artworks and manuscripts, it also applies to scientific discoveries. We believe that the provenance of the delirium phenotype in over $90 \%$ of published articles since 2000 is on or before 1990 . The delirium phenotype in 2015 is remarkably similar to that from $500 \mathrm{BC}$.

In 2014 , the most prolific author on delirium ${ }^{1}$ defended the two major delirium phenotype models, ie, the Diagnostic Statistical Manual for Mental Disorders (DSM) 2 
of the American Psychiatric Association and the Confusion Assessment Method (CAM) ${ }^{3}$ of 1990. Neither the DSM-V nor CAM was built on a logical base in the way Euclid built geometry; rather, they restated prior understanding, consensus, and tradition. Although Inouye et al ${ }^{1}$ claimed that their paper represented "cutting edge", the phenotype was unchanged from 1990 despite advances in Diffuse Lewy body dementia with fluctuations mimicking those in delirium, ${ }^{4}$ behavioral and psychological symptoms of dementia (BPSD) ${ }^{5}$ causing many false positive diagnoses by DSM and CAM, isolated psychosis such as in Parkinson's disease, fluctuating attention in obstructive sleep apnea, and confusional arousals from sleep. ${ }^{6}$ Accurate measurement of fluctuations requires frequent cognitive measurements and behavioral observations, and is not feasible in most hospitals. Inouye et al omitted references and personal communications that undermined their views (logical fallacy of suppressed evidence). ${ }^{7-9}$ Karl Popper, a champion of logic in medicine, advocated bold audacious models and methods which could easily be disproven. Only $1.6 \%$ of key delirium articles $(18 / 1,118)$ used gold standards of speed, amplitude, and criticality of cognitive decline or rapid reversibility. Almost no investigators recognize these self-evident gold standards. The global impression of recovery of delirium in days was reported in 197/1,118 studies (17.6\%) but such impressions are highly subjective and much inferior to regression of cognitive scores to a plateau.

Our deconstruction of 1,118 key delirium articles comprising 7,412 text pages in medical journals (mean year of publication was 2006) convinced us that the models of delirium in the DSM-V, International Classification of Disease (ICD-10), ${ }^{10}$ and CAM ignore an obvious truth, ie, the gold standards of rapid cognitive decline (RCD) are: speed and amplitude of cognitive decline compared with dementia; and speed and amplitude of cognitive improvement in at least $75 \%$ of subjects. Such gold standards apply in airway obstruction, ie, asthma has rapid onset and almost as rapid resolution compared with chronic obstructive pulmonary disease (COPD), which has a very slow progression. When delirium is caused by a relatively irreversible process such as a large middle cerebral artery infarction, reversibility is partial and slower than if delirium is caused by acute respiratory failure which can be corrected within hours by noninvasive ventilation.

The ideal RCD model should contain: universally acceptable assumptions; highly specific definitions that exclude diffuse Lewy body dementia, BPSD, and psychosis without other cognitive changes; and step-by-step logic. This paper provides the first logic-based alternative to delirium orthodoxy. Our model unifies all RCD phenotypes based on cognitive domains, which is an obvious strategy never attempted. DSM-V and CAM in our view rest on logical fallacies, such as appeal to unqualified authority (American Psychiatric Association) and circular reasoning (poor hearing generates false positives). Only 23/1,118 key delirium articles $(2.0 \%)$ tested hearing and $13 / 23$ articles testing hearing $(56.5 \%)$ were from us.

A mathematician observing elderly subjects with cognitive decline would formulate a simple robust classification, ie, speed of onset. Speed of onset distinguishes many disease pairs besides asthma/COPD. Rise in serum creatinine is meteoric in acute renal failure but snail-paced in chronic kidney disease. Slow-onset neurocognitive decline in DSM-V fits this phenotype: slow low-amplitude, noncritical, nonreversible cognitive decline (SLANCNRCD). Cognitive decline 1\%-2\% per 90 days in one or more cognitive domains such as executive function. Noncritical refers to an insignificant impact in 90 days (eg, baseline letter fluency 18 per 60 seconds dropping to 17 words is noncritical, whereas a drop to five words would be critical). SLANCNRCD corresponds to the DSM-V ${ }^{3}$ major minor neurocognitive disorder, formerly labeled as dementia and mild cognitive impairment. Five studies with 2,249 dementia subjects had an annual Mini-Mental State Examination (MMSE) decline of $4.04 \% \pm 2.21 \%{ }^{11-15}$

The second group (RCD) develops 2,300-3,000-fold faster than SLANCNRCD and is highly reversible: rapid high amplitude critical reversible cognitive decline. We selected a $25 \%$ per 24 hours decline in one or more cognitive domains as the cutoff, but future multicenter trials may set a different limit or extend this dichotomy ( $1 \%-24 \%$ versus $\geq 25 \%$ ) into 3-4 categories. The choice of $25 \%$ per 24 hours rests on a similar logic to setting a 1.5 standard deviation (SD) below the age-adjusted mean for cognitive domain scores to define mild cognitive impairment and 2.0 SD for dementia. We have never read convincing proof that $2.0 \mathrm{SD}$ is superior to 2.2 for dementia or that $1.5 \mathrm{SD}$ for mild cognitive impairment is superior to $1.7 \mathrm{SD}$. In a memory clinic, relatively few scores decline by more than $15 \%$ over 6 months, so a decline of $25 \%$ over 24 hours will never be mistaken for fluctuating dementia. Critical decline implies an inability to perform a usual task, such as driving or using a microwave oven. DSM-V lists only one form of RCD, ie, a delirium phenotype with onset in hours to several days. This onset is so broad that clinicians are prone to label any cognitive decline as rapid.

The periodic table in chemistry arranges elements by atomic mass and serves as a "Rosetta Stone" to unlock the secrets of chemistry. Neuropsychologists use a periodic table approach to dementia by measuring each cognitive domain separately. Neuropsychologists work almost only in subacute 
and chronic phase (eg, 2-6 months after stroke). Geriatricians and others assess RCD with multidomain cognitive scales that obscure the individual domain trajectories over time. Our review of 1,118 delirium papers failed to find any work in which neuropsychologists assessed multiple cognitive domains of RCD in the emergency department. Based on this study, we have implemented a 5-minute non-copyrighted paper pencil test battery in the emergency department that does not require digital devices, Internet access, or electricity to assess RCD in five cognitive domains: attention, word-list recall at 5 minutes, language, executive function, and visuospatial function. Transient global amnesia represents RCD in memory/orientation. Focal ischemic stroke causes three RCD phenotypes, ie, language, executive function, and visuospatial function. Delirium in RCD involves mainly attention, memory, and executive function, with lesser impairments in language and visuospatial function.

A sample of 1,646 patients in a Swiss memory clinic had a neuropsychological assessment with 18 tests. ${ }^{16}$ Factor analysis disclosed seven clusters: verbal attention, measured by digit span forward (DSF) and digit span backwards; verbal learning and memory, measured by tests such as word-list recall after 5 minutes; verbal language production (animal category fluency, letter fluency); executive motor function, measured by Trail Making Tests A and B; visual learning and memory; visual attention, measured by Corsi block tests forward and backward; and executive visual function, measured by Stroop tests. Although this Swiss study involves stable patients in a memory clinic, the approach to testing each cognitive domain with brief non-copyrighted bedside tests applies to RCD.

This article excludes rapid, high-amplitude, critical, reversible cognitive decline after general anesthesia or surgery, cardiac arrest, multiple trauma, a stay in intensive care, and subjects with developmental delay. In writing this article, we follow on from many great scientists, including Carl Linnaeus, who in 1735 published "Systema Natura", which divided plants into 24 classes based solely on their sexual organs, and Gregor Mendel, who published his first genetics paper in 1865 and whose ground-breaking model was not recognized for 35 years.

\section{Materials and methods Inclusion and exclusion criteria for CADIS}

Inclusion criteria for CADIS (Central Coast Australia Delirium Intervention Study) were age $\geq 65$ years and admission to Wyong Hospital with prevalent delirium onset within 5 days by four criteria:
- CAM score 20-30/30 or subsyndromal delirium (CAM $15 / 30)$

- Clear proof that elements of CAM, ie, "confusion" and "disorganized thinking", could not be better explained by BPSD. For example, if episodes of disordered behavior had occurred five times in the past 10 days, this would suggest BPSD rather than delirium

- Proof of recent inattention on DSF or by history from informant

- Evidence in the emergency department that inattention, memory impairment, and executive dysfunction were not due to sedatives or antipsychotics.

Many CAM-positive subjects did not meet our three additional criteria and were excluded. We had to base our study on the CAM because this instrument is so entrenched and an initial study could not abandon the CAM, whereas future studies would have support to break free of the CAM. We screened as many emergency department patients as possible, particularly those identified by emergency department staff as confused.

Exclusion criteria were:

- Onset more than 5 days before admission, uncertain date of onset, or incident delirium

- No family, friend, or informant to provide consent

- Not English-speaking

- Admission to orthopedics, surgery, intensive care unit, high dependency unit, coronary care unit, or thrombolysis of acute ischemic stroke

- End-stage dementia or total dependency (7/7) on the Global Deterioration Scale ${ }^{17}$

- End-stage renal failure not on dialysis or recurrent hepatic encephalopathy

- Terminal/palliative care for a condition other than delirium

- Severe visual impairment despite spectacles or magnifying glass not corrected by bold four-fold magnified drawing of dual pentagons for MMSE, ${ }^{18}$ cube copying, clock drawing, and Trail Making Part B in the Montreal Cognitive Assessment ${ }^{19}$ and line drawings on the Addenbrooke Cognitive Examination-Revised ${ }^{20}$

- Severe hearing impairment not corrected with a combination of hearing aids and a portable amplifier with headphones

- Aphasia.

\section{Randomization}

Randomization was by blocks for four from a random numbers table. The trial is registered at ClinicalTrials.Gov with the identifier NCT01650896. 


\section{Demographic and functional measures at baseline}

Education for over $90 \%$ of subjects was 8.9 years to time of leaving high school; less than $5 \%$ had tertiary education. The Informant Questionnaire on Cognitive Decline in the Elderly (IQCODE $)^{21}$ score ranged from 0 to 5. Instrumental Activities of Daily Living (IADL, using the Nottingham scale) $)^{22}$ ranged from 0 (totally dependent) to 22 (independent in activities such as driving, making coffee, cooking, writing letters or emails, laundry). The informant rated IADL pre-delirium and at months 1,3 , and 12 post-discharge.

We created a selective IADL (SIADL) from 8 of 22 Nottingham IADL items not requiring independent mobility. The SIADL ranges from 0 (worst) to 8 (best). The percentage change in SIADL from pre-admission to admission was calculated as 100× (baseline SIADL - admission SIADL)/8.

Carers rated the Apathy Evaluation Scale ${ }^{23}$ (AES) prior to delirium and on admission. AES scores ranged from 18 (severe apathy) to 72 (high motivation): range 54 points. The percentage change in AES from pre-admission to admission was calculated as $100 \times$ (baseline AES - admission AES)/54. We defined systemic active cancer as nonskin cancer that was metastatic or had the potential for dangerous spread. Localized breast cancer treated only by lumpectomy would not qualify. We measured comorbidity with the Charlson Comorbidity Index ${ }^{24}$ and the Cumulative Illness Rating Scale. ${ }^{25}$

\section{General approach to all cognitive testing}

First we ensured patient safety and comfort. Examples of discomfort include pain, nausea, need for toileting, or request to nap. When the patient was safe and comfortable, we inserted hearing aids and performed the whisper test with two numbers and one letter such as: "D 97 ". If the whisper test was abnormal, we obtained verbal consent to place headphones with a portable amplifier (cost US\$120, consumables two AA batteries per year). No subject refused the headphones.

First, we tested attention over 5 seconds as the best of three trials. We used different digit sequences for each trial. The five-digit span forward (5-DSF) has a range from 0 (complete inattention) to five (full attention). The percentage change in 5-DSF from pre-admission to admission was calculated as $100 \times$ (baseline 5-DSF - admission 5-DSF)/5. Six-digit span forward (6-DSF) had a range from 0 (complete inattention) to six (full attention). The percentage change in 6-DSF from pre-admission to admission was calculated as $100 \times$ (baseline 6-DSF - admission 6-DSF)/6. The 5-DSF is often normal in subjects with mild inattention, so addition of 6-DSF testing was essential.
The MMSE, ${ }^{18}$ Montreal Cognitive Assessment, ${ }^{19}$ and Addenbrooke Cognitive Examination-Revised ${ }^{20}$ (after discharge) used bold, four-fold enlarged drawings when the subject had visual impairment.

The Delirium Index ${ }^{26}$ (DI) has a range of 0 (best possible score) to 21 (severe cognitive, psychomotor, and psychotic features). The percentage change in DI from pre-admission to admission was calculated as $100 \times$ (baseline DI - admission D1)/21.

Improvements in 5-DSF, 6-DSF, and DI during first 14 days in hospital were based on the best score after admission. We then calculated maximal possible change as (initial value - maximal value)/range of scale. Next, we calculated actual change/maximal possible change ratio. Example: initial 5-DSF $=1$; maximal possible change $(5-1) / 5=80 \%$; best value during hospital stay is 4 , so actual change $4-1=3 / 5$ (60\%); actual change/maximal possible change ratio is $60 / 80=75 \%$. Had the 5 -DSF improved from $0 / 5$ to $5 / 5$, this ratio would be $100 \%$.

\section{Imputing DSF, DI, and MMSE prior to delirium}

Many of the subjects who attended the Wyong Hospital memory clinic had a recent measurement of DSF, DI, and MMSE. Others had MMSE alone within months of admission. We defined recent as 1-6 months unless there was a brain event such as stroke after the most recent test. Imputing a pre-illness cognitive score is defined as a best estimate from recent scores or from a score after recovery. We label using recent scores as forward imputation and using recovery scores as reverse imputation. Reverse imputation is illustrated by an initial DI of $14 / 21$ improving to $0 / 21$ by the time of discharge. We reverse impute pre-delirium DI as $0 / 21$. For every patient with forward imputation data, there were three with only reverse imputation figures.

\section{Daily monitoring of digit span and $\mathrm{DI}$}

CADIS is one of the less than $1.3 \%(15 / 1,118)$ of delirium articles monitoring attention, orientation, psychosis, and three-word recall daily 365 days per year. We monitored until either hospital discharge, resolution of score, or hospital day 14. It was impossible to blind the observer making daily measurements because the ward location disclosed treatment group. After discharge, WP assessed subjects blinded to treatment group but those measurements are in a subsequent report.

We used Stats Direct version 2.7.9 9 (July 2012) for descriptive statistics, and the Chi-square test, Fisher's Exact test, and Wilcoxon signed rank test to analyze improvement in test scores over time and for comparing between two groups. The Northern Sydney Research Ethics Committee 
approved the study, and informed consent was provided by relatives or legal guardians.

\section{Results}

We recruited 130 patients from July 16, 2012 to May 14, 2014 (ie, 22 months). We excluded 14 subjects with subsyndromal delirium, leaving 116 subjects with delirium by CAM and our three other stringent diagnostic criteria. The 116 patients with emergency room-prevalent delirium were of mean age 83.6 \pm 7.4 years (Table 1). Sixty-six $(57.0 \%)$ were female and $94(81.0 \%)$ were community-dwelling. Twenty-three $(24.5 \%)$ of the 94 community-dwellers lived alone. Twentyfive subjects $(21.6 \%)$ were drivers; $43.1 \%$ were currently married and $50.9 \%$ were widowed. Mean duration of education was $8.9 \pm 2.2$ years. The IQCODE score was $3.76 \pm 0.66$. Fifty-six patients $(48.3 \%)$ had a prior specialist diagnosis of dementia, and $28(24.1 \%)$ were diabetic. According to family and informants, the mean interval between delirium onset and admission was $1.31 \pm 1.36$ (range $0-5$ ) days. The mean IADL score before delirium was 9.6 \pm 6.8 . Only $17 \%$ lacked a $25 \%$ acute decline in 5-DSF or 6-DSF. Had we repeated these tests twice at hourly intervals in the emergency room, the proportion without critical DSF decline would have been less.

\section{Acuity and magnitude of cognitive decline}

The mean SIADL score before delirium was $4.11 \pm 2.39$ and decreased to $0.80 \pm 1.44$ on admission (Table 1, relative change $[\mathrm{RC}] /$ decline $41.4 \%$ in executive function through activities, $P<0.001)$. The mean MMSE score was $24.4 \pm 4.6$ before delirium and decreased to $11.4 \pm 6.9(\mathrm{RC}-42.3 \%, P<0.001)$. The DI before delirium was $2.13 \pm 1.96$ ( 0 is best score) and worsened to $11.5 \pm 3.3$ on admission ( $\mathrm{RC}-44.3 \%, P<0.0001$ ). The mean 5-DSF before delirium was $4.99 \pm 0.09$ and decreased to $3.28 \pm 2.13$ on admission ( $\mathrm{RC}-34.0 \%, P<0.001$ ). 6-DSF before delirium was $5.54 \pm 0.97$ and decreased to $1.84 \pm 2.19$ on admission $(\mathrm{RC}-53.8 \%, P<0.0001)$. Mean AES before delirium was $45.3 \pm 14.2$ and decreased to $25.4 \pm 9.9$ on admission (Table 1 , RC $-36.7 \%, P<0.001)$. Combining all six tests and weighting them equally mean RC was $43.2 \%$ (lowest $\mathrm{RC}-34.0 \%$ for 5 -DSF to highest RC $60.8 \%$ for 6-DSF). Univariate analysis of change for each parameter were significant at the $P<0.0001$ level. Hospital mortality was 10/116 (Table 1, 8.6\%).

\section{Rapidity of improvement}

Patients who survived or died were censored to the best score after admission. 5-DSF improved by $31.7 \%$ compared with the maximal possible improvement of $32.4 \%$ (Table 2 ). Actual improvement was $97.8 \%$ of maximal possible gain. 6-DSF improved by $53.8 \%$ compared with a maximal possible
Table I Pre-delirium versus admission characteristics of II6 patients with prevalent delirium

\begin{tabular}{|c|c|}
\hline Feature & Mean \pm SD or proportion \\
\hline Age, years & $83.6 \pm 7.4$ \\
\hline Female & $66 / 116(56.9 \%)$ \\
\hline Community-dwelling & $94 / 116(81.0 \%)$ \\
\hline Home alone/community dwelling & $23 / 94(24.5 \%)$ \\
\hline Education years & $8.9 \pm 2.2$ \\
\hline IQCODE score (0-5) & $3.76 \pm 0.66$ \\
\hline Dementia prior to admission & $56 / 116(48.3 \%)$ \\
\hline CIRS (comorbidity) & $7.4 I \pm 2.34$ \\
\hline Charlson Comorbidity Index & $3.87 \pm 1.76$ \\
\hline Weight, kg & $70.6 \pm 16.4$ \\
\hline Body mass index & $26.9 \pm 5.1$ \\
\hline LOS (mean/median) & $18.5 \pm 20.6 / 12.0$ \\
\hline IADL pre-delirium ( 0 , worst; 22 , best $)$ & $9.6 \pm 6.8$ \\
\hline SIADL pre-delirium ( 0 , worst; 8 , best) & $4.11 \pm 2.39$ \\
\hline SIADL admission/RC \% & $0.80 \pm 1.44 /-4 \mid .4 \%$ \\
\hline MMSE pre-delirium & $24.4 \pm 4.6$ \\
\hline MMSE admission/RC \% & |l. $.4 \pm 6.9 /-42.3 \%$ \\
\hline DI pre-delirium & $2.13 \pm 1.96$ \\
\hline DI admission/RC \% & II. $5 \pm 3.3 /-44.3 \%$ \\
\hline 5-DSF pre-delirium & $4.99 \pm 0.09$ \\
\hline 5-DSF admission/RC \% & $3.28 \pm 2.13 /-34.0 \%$ \\
\hline 6-DSF pre-delirium & $5.54 \pm 0.97$ \\
\hline 6-DSF admission/RC \% & $1.84 \pm 2.19 /-53.8 \%$ \\
\hline Apathy pre-delirium on AES & $45.3 \pm 14.2$ \\
\hline AES admission/RC \% & $25.4 \pm 9.9 /-36.7 \%$ \\
\hline
\end{tabular}

Notes: The data are shown as the mean $\pm S D$. RC is the relative change predelirium to admission as a percentage and is calculated as RC $=100 \times$ absolute change/range of test.

Abbreviations: 5-DSF, five-digit span forward; 6-DSF, six-digit span forward; AES, Apathy Evaluation Scale; CIRS, Cumulative Illness Rating Scale; DI, Delirium Index; IADL, Instrumental Activities of Daily Living; IQCODE, Informant Questionnaire on Cognitive Decline in the Elderly; LOS, length of stay; MMSE, Mini-Mental State Examination; RC, relative change; SD, standard deviation; SIADL, Selective Instrumental Activities of Daily Living.

improvement of $65.8 \%$ (Table 2). Actual improvement was $81.8 \%$ of maximal possible gain. DI improved by $36.4 \%$ compared with maximal possible improvement of $45.2 \%$ (Table 2). Actual improvement was $80.5 \%$ of maximal possible gain. SIADL improved by $19.2 \%$ compared with maximal possible improvement of $41.4 \%$ (Table 2). Actual improvement was $46.4 \%$ of maximal possible gain. AES and MMSE were not measured after baseline until 1 month postdischarge, so no figures are provided here for improvement during hospital stay. Combining all four tests (5-DSF, 6-DSF, DI, and SIADL) the mean relative improvement was $35.3 \%$ (low of $19.2 \%$ for SIADL to a high of $53.8 \%$ for $6-\mathrm{DSF}$ ). The mean actual/maximal improvement ratio was $76.6 \%$. The mean $\pm \mathrm{SD}$ days for recovery was $2.00 \pm 3.79$ for the

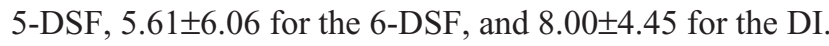
Univariate analysis of improvement for each parameter (eg, 6-DSF or DI) were significant at the $P<0.0001$ level. 
Table 2 Comparison of admission versus best or discharge features for all I 16 subjects

\begin{tabular}{ll}
\hline Feature & Mean \pm SD or \% \\
\hline Improvement 5-DSF \% & $31.7 \pm 42.2$ \\
Maximum possible change \% & 32.4 \\
Actual change/maximum possible change & 97.8 \\
Days to restoring 5-DSF & $2.00 \pm 3.79$ \\
Improvement in 6-DSF \% & $53.8 \pm 38.8$ \\
Maximum possible change \% & 65.8 \\
Actual change/maximum possible change & 81.8 \\
Days to restoring 6-DSF & $5.61 \pm 6.06$ \\
Improvement in DI \% & $36.4 \pm 19.2$ \\
Maximum possible change \% & 45.2 \\
Actual change/maximum possible change & 80.5 \\
Days to restoring DI & $8.00 \pm 4.45$ \\
Improvement in SIADL \% & 19.2 \\
Maximum possible change \% & 41.4 \\
Actual change/maximum possible change & 46.4 \\
Died in hospital & $10 / I 16$ (8.6\%) \\
Discharge to nursing home & $27 / I 16$ (23.3\%) \\
\hline Notes: The data are shown as the mean \pm SD. Maximum possible change would be \\
I00\% if the initial mean score was 0/5 and the mean discharge score was 5/5. If the \\
initial mean was 3/5, the maximum possible change would be 40\%. \\
Abbreviations: 5-DSF, five-digit span forward; 6-DSF, six-digit span forward; \\
DI, Delirium Index; SD, standard deviation; SIADL, Selective Instrumental Activities \\
of Daily Living.
\end{tabular}

\section{Comparison of randomized groups}

Tables 3-5 compare elderly patients with delirium randomized to general medicine $(\mathrm{n}=58)$ or to geriatric medicine $(\mathrm{n}=58)$. The two groups were similar in age, sex, community-dwelling status, living alone, educational level, IQCODE, dementia before delirium, AES, Cumulative Illness Rating Scale score, Charlson Comorbidity Index, weight, and body mass index (Table 3). IADL pre-delirium was 3.0 points higher in the medical group (relative difference $13.6 \%$ or $3 / 22$ ) but the $P$-value of 0.032 became insignificant after Bonferroni correction for 15 baseline comparisons. Pre-delirium SIADL was 0.47 higher in the medical group (relative difference $5.9 \%$, not statistically significant). Univariate analysis of change within each group for parameters monitored daily (eg, 6-DSF or DI) was significant at the $P<0.0001$ level.

Table 4 shows that the decreases from pre-delirium to admission in 5-DSF, 6-DSF, MMSE, DI, SIADL, and AES were comparable in the medical and geriatric medical groups (the mean of ten $P$-values being 0.60). Likewise combining three, four, or five of those measures did not lead to significant differences between groups (Table 4).

Table 5 shows the improvements in each delirium marker during the hospital stay. Univariate analysis of improvement for DSF and DI was significant at the $P<0.0001$ level. Although 6-DSF improved more in the geriatric medical group than in the medical group $(P=0.078)$ after Bonferroni correction, this $P$-value became insignificant (0.312). The
Table 3 Comparison of pre-delirium features of medical and geriatric medical groups each with 58 subjects

\begin{tabular}{llll}
\hline Feature & Medical & $\begin{array}{l}\text { Geriatric } \\
\text { medical }\end{array}$ & P-value \\
\hline Age, years & $83.0 \pm 7.7$ & $84.3 \pm 7.5$ & $0.5 \mathrm{I}$ \\
Female & $33 / 58(56.9 \%)$ & $33 / 58(56.9 \%)$ & 0.99 \\
Community-dwelling & $45 / 58(77.6 \%)$ & $50 / 58(86.2 \%)$ & 0.24 \\
Home alone/community & $14 / 45(31.1 \%)$ & $9 / 50(18.0 \%)$ & 0.15 \\
dwelling & & & \\
Years of education & $9.0 \pm 2.1$ & $8.7 \pm 2.3$ & 0.41 \\
IQCODE & $3.64 \pm 0.61$ & $3.87 \pm 0.69$ & $0.075(\mathrm{NS})$ \\
$\begin{array}{l}\text { Dementia prior to } \\
\text { admission }\end{array}$ & $25 / 58(43.1 \%)$ & $31 / 58(53.4 \%)$ & 0.27 \\
Systemic active cancer & $8 / 58(13.8 \%)$ & $1 / 58(1.7 \%)$ & 0.017 \\
IADL pre-delirium (0-22) & $11.1 \pm 7.3$ & $8.1 \pm 6.1$ & 0.032 \\
SIADL pre-delirium (0-8) & $4.35 \pm 2.44$ & $3.88 \pm 2.33$ & 0.19 \\
Apathy pre-delirium on & $47.7 \pm 14.0$ & $43.4 \pm 14.0$ & 0.19 \\
AES & & & \\
CIRS (comorbidity) & $7.21 \pm 2.46$ & $7.62 \pm 2.21$ & 0.30 \\
Charlson Comorbidity & $3.88 \pm 1.85$ & $3.86 \pm 1.62$ & 0.80 \\
Index & & & \\
Weight kg & $70.7 \pm 17.7$ & $70.5 \pm 15.3$ & 0.95 \\
Body mass index & $27.4 \pm 5.6$ & $26.5 \pm 4.7$ & 0.321 \\
\% first I4 hospital days in & $23.8 \pm 42.3$ & $83.9 \pm 33.8$ & $<0.000 \mathrm{I}$ \\
GU versus other wards & & & \\
\hline
\end{tabular}

Notes: The data are shown as the mean \pm SD. After Bonferonni correction for 15 comparisons, none of the $P$-values were significant except for hospital days in the geriatric unit, which was directed by randomization.

Abbreviations: AES, Apathy Evaluation Scale; CIRS, Cumulative Illness Rating Scale; IADL, Instrumental Activities of Daily Living; IQCODE, Informant Questionnaire on Cognitive Decline in the Elderly; SD, standard deviation; SIADL, Selective Instrumental Activities of Daily Living; GU, geriatric unit; NS, not statistically significant.

Table 4 Comparison of admission versus pre-admission features of medical and geriatric medical groups each with 58 subjects

\begin{tabular}{llll}
\hline Feature & $\begin{array}{l}\text { Medical } \\
\text { group }\end{array}$ & $\begin{array}{l}\text { Geriatric } \\
\text { medical }\end{array}$ & P-value \\
\hline 5-DSF on admission & $3.33 \pm 2.09$ & $3.24 \pm 2.19$ & 0.93 \\
\% change in 5-DSF & $33.4 \pm 41.8$ & $34.6 \pm 43.5$ & 0.95 \\
6-DSF on admission & $2.18 \pm 2.42$ & $1.51 \pm 1.91$ & 0.22 \\
\% change in 6-DSF & $58.1 \pm 39.2$ & $63.4 \pm 31.0$ & 0.65 \\
MMSE on admission & $12.0 \pm 6.9$ & $10.9 \pm 7.0$ & 0.37 \\
\% change in MMSE & $42.8 \pm 24.1$ & $41.9 \pm 26.3$ & 0.76 \\
DI on admission & $11.1 \pm 3.2$ & $11.9 \pm 3.4$ & 0.176 \\
\% change in 5-DI & $43.5 \pm 16.6$ & $45.0 \pm 17.4$ & 0.69 \\
\% change SIADL & $41.0 \pm 26.3$ & $41.7 \pm 29.6$ & 0.93 \\
\% change AES & $39.2 \pm 24.2$ & $34.2 \pm 23.8$ & 0.37 \\
Mean \% $\Delta$ 5DSF/6DSF/DI & $44.5 \pm 27.2$ & $47.3 \pm 24.8$ & 0.47 \\
Mean \% $\Delta$ 5DSF/6DSF/ & $43.5 \pm 22.5$ & $45.9 \pm 21.9$ & 0.62 \\
DI/SIADL & & & \\
Mean \% $\Delta$ 5DSF/6DSF/ & $42.4 \pm 19.3$ & $43.9 \pm 19.2$ & 0.82 \\
DI/SIADL/AES & & & \\
\hline Notes: & &
\end{tabular}

Notes: The data are shown as the mean \pm SD. Pre-admission scores were imputed. Abbreviations: 5-DSF, five-digit span forward; 6-DSF, six-digit span forward; AES, Apathy Evaluation Scale; DI, Delirium Index; MMSE, Mini-Mental State Examination; SD, standard deviation; SIADL, Selective Instrumental Activities of Daily Living. 
Table 5 Comparison of admission versus best or discharge features of medical and geriatric medical groups, each with 58 subjects

\begin{tabular}{|c|c|c|c|}
\hline Feature & Medical & $\begin{array}{l}\text { Geriatric } \\
\text { medical }\end{array}$ & $P$-value \\
\hline Improvement 5-DSF \% & $30.0 \pm 41.2$ & $33.4 \pm 43.9$ & 0.77 \\
\hline Maximum possible change $\%$ & 33.4 & 35.2 & \\
\hline $\begin{array}{l}\text { Actual change/maximum } \\
\text { possible change }\end{array}$ & 90.0 & 95.0 & \\
\hline Improvement 6-DSF \% & $47.3 \pm 41.0$ & $60.2 \pm 35.7$ & 0.078 \\
\hline Maximum possible change $\%$ & 63.7 & 74.8 & \\
\hline $\begin{array}{l}\text { Actual change/maximum } \\
\text { possible change }\end{array}$ & 74.2 & 80.5 & \\
\hline Improvement DI \% & $36.0 \pm 18.2$ & $36.9 \pm 20.4$ & 0.93 \\
\hline Maximum possible change $\%$ & 47.1 & 43.3 & \\
\hline $\begin{array}{l}\text { Actual change/maximum } \\
\text { possible change }\end{array}$ & 76.4 & 85.2 & \\
\hline $\begin{array}{l}\text { Improvement in SIADL \% } \\
\text { change }\end{array}$ & $19.6 \pm 22.5$ & $18.7 \pm 21.6$ & 0.86 \\
\hline Maximum possible change & 41.5 & $4 I .1$ & \\
\hline $\begin{array}{l}\text { Actual change/maximum } \\
\text { possible change }\end{array}$ & 47.2 & 45.5 & \\
\hline Mean LOS & $16.1 \pm 16.5$ & $20.8 \pm 24.0$ & 0.039 \\
\hline LOS median & 9.5 & 14.0 & \\
\hline Died on discharge & $5 / 58(8.6 \%)$ & $5 / 58(8.6 \%)$ & 0.99 \\
\hline Discharged to nursing home & I5/58 (25.9\%) & I2/58 (20.7\%) & 0.70 \\
\hline Mean days of follow-up & $3|8.5 \pm 2| 4.2$ & $3|I .1 \pm 2| 4.0$ & 0.86 \\
\hline
\end{tabular}

Note: The data are shown as the mean \pm SD.

Abbreviations: 5-DSF, five-digit span forward; 6-DSF, six-digit span forward DI, Delirium Index; LOS, length of stay; SD, standard deviation; SIADL, Selective Instrumental Activities of Daily Living.

mean length of stay was 4.7 days longer in the geriatric medical group than in the medical group (relative difference $29.1 \%, P=0.039$ ) and the median length of stay was 4.5 days longer (relative difference $47.3 \%$ ). This most likely represents the tendency for general medical units to discharge patients on completion of treatment, whereas geriatric units use functional improvement criteria to determine suitability for discharge. Assume a medical group patient spends 9/14 hospital days on the medical ward and 5 days in the geriatric unit, the amount of time spent in the geriatric unit would be $5 / 14$ days (36\%). Discharge mortality ( $8.6 \%)$ and proportions discharged to nursing homes were similar (Table 5).

\section{Discussion}

The 116 subjects in this study were identified in the emergency department to have prevalent delirium by four criteria: CAM, proof that confusion and disorganized thinking were not better explained by BPSD, proof of recent $25 \%$ or greater decline in DSF, and evidence that cognitive changes could not be attributed to sedatives or antipsychotics. This phenotype behaved exactly like a rapid onset and almost as rapidly reversible condition such as asthma compared with the slowly progressive airway obstruction in COPD. The mean decline in six scores from pre-delirium to admission was $43.2 \%$ and the mean improvement in the first 14 days was $35.3 \%$. We concede that many patients had variable and fluctuating scores, but the most logical way to measure improvement was to select the best score during daily follow-up. The fact that $48 \%$ of our subjects had a firm clinical diagnosis of dementia pre-delirium by a geriatrician, neurologist, or psychiatrist shows the similarity of the analogy with COPD versus asthma. When dementia has a behavioral flare without $\mathrm{RCD}$, the correct label is BPSD. When cognition in dementia patients declines by $25 \%$ over 24 hours, RCD is present.

Functional Independence Measure efficiency gains in stroke rehabilitation parallel the improvements we report in delirium recovery, but these gains are likely to be more stable for periods of 1 week than cognitive scores in recovering delirium.

Our criteria for diagnosis was associated with greater cognitive improvement than in a Singapore study of 234 elderly patients admitted to a five-bed delirium unit ${ }^{27}$ in which the DRS-98 severity subscale improved by 7.51 points on a 39-point scale during a mean 3.8 days in the delirium unit (RC $7.51 / 39=19.2$ ); the Chinese MMSE improved by 3.44 points on a 28-point scale ( $\mathrm{RC} 3.44 / 28=12.3 \%$ ); and the modified Barthel ADL improved by 19.18 points on a 100-point scale (RC 19.2\%). The authors reported that $28.6 \%$ of their 234 subjects had BPSD prior to delirium (less than $2 \%$ of delirium articles reported on BPSD). The DRS-R98 improvement in Singapore (19.8\%) was only $53 \%$ as great as the DI improvement in our study.

Our approach to RCD resembles Koch's postulates for demonstrating a causative link between a pathogen and a disease. Here is a way of restating RCD in postulates like Koch. The first postulate: medical history from family, reliable informants, and health professionals to establish a $25 \%$ cognitive decline over 24 hours and rule out other phenotypes such as BPSD (where behavioral change exceeds cognitive decline) and isolated psychosis (delusions, hallucinations, and thought disorder). Second tests for each cognitive domain isolate which to Second postulate: test each cognitive domain to isolate which domain(s) is mainly affected. Third monitor cognitive domains to Third postulate: monitor cognitive domains daily to assess rate of improvement unless either the patient dies or has a new irreversible brain disorder (eg, large-volume ischemic stroke).

Those who argue that a focus on cognitive scores misses the big picture should consider the system of plant taxonomy introduced by Carl Linnaeus in 1735 in "Systema Natura". This system, which classified plants exclusively by their sexual organs, remains valid 279 years later. Opening the door to behavioral changes has led to false-positive diagnoses of delirium in BPSD. 


\section{Conclusion}

The delirium phenotype in this article is unique among 1,118 key delirium articles. It separates delirium from dementia in the way asthma is distinguished from COPD: speed on onset and speed of recovery. The issue of delirium superimposed on dementia resembles an exacerbation of COPD. The 1,118 delirium articles focus on underdiagnosis of delirium whereas overdiagnosis of delirium is more important because it abandons medical logic. We suggest that the limited progress in delirium science is due to using a primitive phenotype which routinely counts many BPSD presentations as delirium.

\section{Acknowledgments}

The author is grateful to Aileen Carter, research occupational therapist, for 2 years of meticulous assessment, and to Wade Penn and Jamie Gills, clinical nurse consultants. These data were presented in part at the October 2013 and October 2014 annual scientific meetings of the International Psychogeriatric Association.

\section{Disclosure}

The author reports no conflicts of interest in this work.

\section{References}

1. Inouye SK, Westendorp RG, Saczynski JS. Delirium in elderly people. Lancet. 2014;383:911-922.

2. American Psychiatric Association. Diagnostic and Statistical Manual for Mental Disorders Fifth Edition. Washington, DC, USA: American Psychiatric Association; 2013.

3. Inouye SK, van Dyck CH, Alessi CA, Balkin S, Siegal AP, Horwitz RI. Clarifying confusion: the Confusion Assessment Method. A new method for detection of delirium. Ann Intern Med. 1990;113:941-948.

4. Vardy E, Holt R, Gerhard A, Richardson A, Snowden J, Neary D. History of a suspected delirium is more common in dementia with Lewy bodies than Alzheimer's disease: a retrospective study. Int J Geriatr Psychiatry. 2014;29:178-181.

5. Landreville P, Voyer P, Carmichael PH. Relationship between delirium and behavioral symptoms of dementia. Int Psychogeriatr. 2013; 25:635-643.

6. Ohayon MM, Mahowald MW, Leger D. Are confusional arousals pathological? Neurology. 2014;83:834-841.

7. Regal P. Confusion Assessment Method indicators when CAM positivity in 647 patients has good outcome. J Am Geriatr Soc. 2013;61:173.

8. Regal P, Hetherington E, Nair B. Delirium Index six-monthly in patients with dementia, mild cognitive impairment and subjective cognitive impairment: keys to interpreting Delirium Index in cognitive impairment. J Neurol Res. 2013;3:96-100.

Clinical Interventions in Aging

\section{Publish your work in this journal}

Clinical Interventions in Aging is an international, peer-reviewed journal focusing on evidence-based reports on the value or lack thereof of treatments intended to prevent or delay the onset of maladaptive correlates of aging in human beings. This journal is indexed on PubMed Central, MedLine,
9. Regal P. Need for new methods to study delirium. Intern Med J. 2013; 43:1053-1054.

10. World Health Organization. ICD-10 International Classification of Mental and Behavioural Disorders. 10th Revision. 4th ed. Geneva, Switzerland: World Health Organization; 2011.

11. Carmichael O, Schwarz C, Drucker D, et al; Alzheimer's Disease Neuroimaging Initiative. Longitudinal changes in white matter disease and cognition in the first year of the Alzheimer disease neuroimaging initiative. Arch Neurol. 2010;67:1370-1378.

12. Bruandet A, Bombois S, Maurage CA, et al. Alzheimer disease with cerebrovascular disease and vascular dementia: clinical features and course compared with Alzheimer disease. J Neurol Neurosurg Psychiatry. 2009;80:133-140.

13. Miekle MM, Rosenberg PB, Tschanz J, et al. Vascular factors predict rate of progression in Alzheimer disease. Neurology. 2007;69:1850-1858.

14. Monaci L, Morris RG. Neuropsychological screening performance and the association with activities of daily living and instrumental activities of daily living in dementia: baseline and 18- to 24-month follow-up. Int J Geriatr Psychiatry. 2012;27:197-204.

15. Munro CA, Longmire CF, Drye LT, et al; Depression in Alzheimer's Disease Study-2 Research Group. Cognitive outcomes after sertraline treatment in patients with depression of Alzheimer disease. Am J Geriatr Psychiatry. 2012;20:1036-1044.

16. Beck IR, Schmid NS, Berres M, Monsch AU. Establishing robust cognitive dimensions for characterization and differentiation of patients with Alzheimer's disease, mild cognitive impairment, frontotemporal dementia and depression. Int J Geriatr Psychiatry. 2014;29:624-634.

17. Reisberg B, Ferris SH, de Leon MJ, et al. The Global Deterioration Scale for assessment of primary degenerative dementia. Am J Psychiatry. 1982;139:1136-1139.

18. Folstein MF, Folstein SE, McHugh PE. Mini-Mental State. A practical method for grading the cognitive state of patients for the clinician. J Psychiatr Res. 1975;12:189-199.

19. Nasreddine AS, Phillips NA, Bedirian V, et al. The Montreal Cognitive Assessment, MoCA: a brief screening tool for mild cognitive impairment. J Am Geriatr Soc. 2005;53:695-699.

20. Mioshi E, Dawson K, Mitchell J, Arnold R, Hodges JR. The Addenbrooke's Cognitive Examination Revised (ACE-R): a brief cognitive test battery for dementia screening. Int J Geriatr Psychiatry. 2006;21:1078-1085.

21. Jorm AF. A short form of informant questionnaire on cognitive decline in the elderly (IQCODE). Psychol Med. 1994;24:145-153.

22. Nouri FM, Lincoln NB. An extended activities of daily living scale for stroke patients. Clin Rehabil. 1987;1:301-305.

23. Marin RS, Biedrzycki RC, Firinciogullari S. Reliability and validity of the Apathy Evaluation Scale. Psychiatry Res. 1991;38:143-162.

24. Charlson ME, Pompei P, Ales KL, Mackenzie CR. A new method of classifying prognostic comorbidity in longitudinal studies: development and validation. J Chronic Dis. 1987;40:373-383.

25. Linn BS, Linn MW, Gurel L. Cumulative Illness Rating Scale. J Am Geriatr Soc. 1968;16:622-626.

26. McCusker J, Cole M, Bellavance F, Primeau F. The reliability and validity of a new measure of severity of delirium. Int Psychogeriatr. 1998; 10:421-433.

27. Lam Ching-Yu, Tay L, Chan M, Ding YY, Chong MS. Prospective observational study of delirium recovery trajectories and associated short-term outcomes in older adults admitted to a specialized delirium unit. J Am Geriatr Soc. 2014;62:1649-1657.

\section{Dovepress}

CAS, Scopus and the Elsevier Bibliographic databases. The manuscript management system is completely online and includes a very quick and fair peer-review system, which is all easy to use. Visit http://www.dovepress. com/testimonials.php to read real quotes from published authors. 